Laser Chem. 1988, Vol. 9, pp. 155-170

(C) 1988 Harwood Academic Publishers GmbH

Photocopying permitted by license only

Reprints available directly from the Publisher

Printed in the United Kingdom

\title{
Photochemical Chain Reactions in Amorphous Solids
}

\author{
ARTHUR J. SEDLACEK and CHARLES A. WIGHT \\ Department of Chemistry, University of Utah, Salt Lake City, UT 84112
}

(Received January 28, 1988; in final form February 23, 1988)

In this article, we review recent work in the authors' laboratory on the subject of free radical chain reactions of chlorine with small hydrocarbons in amorphous solids. The solids are formed as thin films by spray deposition of the gaseous reagents onto a cryogenic window. Reactions are initiated by excimer laser photolysis at $308 \mathrm{~nm}$, which dissociates a small fraction of the chlorine molecules to atoms. Product yields and branching ratios are determined by infrared absorption spectroscopy. Reactions of chlorine with cyclopropane or cyclobutane proceed by true chain reactions, as evidenced by high product quantum yields (number of product molecules formed per laser photon absorbed by the sample). Measurements of the dependence of the product yield on the relative concentrations of chlorine and hydrocarbon provide clues to the reaction mechanism in the solid state. The cyclobutane reaction appears to involve $\mathrm{H}$ atom transfer from cyclobutane to cyclobutyl radical as an intermediate step in the overall reaction. Reaction of chlorine with propane, $n$-butane, or isobutane does not appear to involve chain propagation and is dominated by radical recombination processes which result in low quantum yields. All of these results are discussed in terms of reactions which occur in a solid state environment where molecular motion is severely restricted.

KEY WORDS: Chain reactions of chlorine with hydrocarbons, amorphous solids, chain propagation, radical recombination.

\section{INTRODUCTION}

Our understanding of solid-state reactivity is presently experiencing a period of remarkable growth and expansion. Most studies have centered on reactions in crystalline solids ${ }^{1,2}$ since their known structure 
provides the investigator with a knowledge of the relative positions between the potential reaction partners. When this information is coupled with spectroscopic detection of the reaction products, it is sometimes possible to determine many of the details of the reaction mechanism. One of the important tasks of chemists is to develop a physical insight to how chemical reactions occur in an environment where molecular motion is highly restricted.

Even though investigations of reactions in crystals can be quite detailed, these studies suffer from limited applicability since most systems of practical interest lack long range order. It is rarely possible to prepare large crystals containing two or more reagents. On the other hand, studies of amorphous solids are burdened by complexities of ill-defined structure which render detailed mechanistic analyses very difficult. In addition, many amorphous solids are unsuitable for spectroscopic interrogation due to a high degree of light scattering or absorption.

Despite the difficulties involved, mechanistic studies of reactions in amorphous solids could be of enormous consequence in many fields. A few examples include relaxation of glasses following photoexcitation, ${ }^{3-5}$ synthesis of new materials with novel, electric, magnetic, or chemical properties, ${ }^{6}$ and polymerization. ${ }^{7,8}$ Some of the features of simple model systems may also be useful for understanding complex reaction mechanisms in biological systems. Karplus has noted that proteins have many of the properties of glasses. ${ }^{9}$ A fundamental understanding of reactions mechanisms in semi-rigid, semi-structured environments will undoubtedly be useful for interpreting experiments in biological systems.

We have initiated a research program to study reactions of free radicals in amorphous films formed by spray deposition of gaseous reagents onto a cryogenic window. ${ }^{10-13}$ This technique allows the samples to be probed by conventional spectroscopic techniques and avoids problems of phase separation which may occur when liquid samples are frozen. Radicals are generated within the solid films by pulsed excimer laser photolysis, and the product yields and branching ratios are determined by obtaining infrared absorption spectra of the films before and after photolysis. Initial experiments have focused on reactions of chlorine with small hydrocarbons. In the gas phase ${ }^{14}$ and in liquid solutions, ${ }^{15}$ these compounds react by free radical chain mechanisms as shown in Reactions (1) and (2). 


$$
\begin{aligned}
& \mathrm{Cl}+\mathrm{RH} \longrightarrow \mathrm{HCl}+\mathrm{R} \cdot \\
& \mathrm{R} \cdot+\mathrm{Cl}_{2} \longrightarrow \mathrm{RCl}+\mathrm{Cl} .
\end{aligned}
$$

Because each elementary step in the mechanism regenerates a radical used in the other step, it is possible for a small concentration of free radicals to convert relatively large amounts of reactants to products. Turnover numbers for these reactions can be as high as $10^{7}$.

Chain reactions make excellent models for studying reaction mechanisms in amorphous solids. The reaction product quantum yields provide a qualitative measure for the ease with which radicals are able to react with their nearest neighbors in the solid. In cases where the turnover numbers (quantum yields) are high, most steps occur away from the original site of photolysis and the influence of excess energy deposited by the laser pulse is minimized. In this limit, the reactions are truly characteristic of the solid environment. Features of the reactions which differ markedly from the well-studied reactions in the gas phase and solution provide clues to the influence of the solid state environment on the details of the reaction mechanism. In addition, careful spectroscopic analysis of the products can sometimes provide highly detailed stereochemical information about the mechanism, as in the case of the chlorine/cyclopropane reaction. ${ }^{10}$

\section{EXPERIMENTAL SECTION}

The experimental techniques are in many ways similar to those of matrix isolation. An optical window (usually CsI for infrared studies or Suprasil quartz for UV absorption) is mounted at the cold tip of a liquid nitrogen dewar vessel, which maintains the temperature at $77 \mathrm{~K}$. In some experiments, a closed cycle helium refrigerator has been used to vary the temperature in the range $10-100 \mathrm{~K}$. The vacuum shroud is equipped with a sample inlet, two CsI windows mounted on opposite sides for obtaining infrared absorption spectra, and one quartz window through which the samples are irradiated with the laser. The sample window can be rotated to face the inlet tube or any of the external windows.

In a typical experiment, a binary mixture of chlorine and the hydrocarbon are mixed together in a glass vacuum manifold. The sample size is typically 0.06 mmoles (a few torr in a 0.3 liter volume). The mole ratio of reactants is typically $1: 1$, but is varied in some 
experiments from about 10:1 to 1:10. Deposition of the sample usually takes $20-60 \mathrm{~min}$ and is carried out in a darkened room to prevent chain reactions from occurring in the inlet system. The entire dewar vessel is placed in the infrared spectrometer (Digilab/Biorad FTS-40) and a spectrum of the frozen film is obtained prior to laser irradiation.

The photolysis is carried out with a $\mathrm{XeCl}$ excimer laser ( $308 \mathrm{~nm}$ ) at relatively low fluence, typically $30-10^{4}$ pulses at $1 \mathrm{~mJ} / \mathrm{cm}^{2} /$ pulse. Low fluence conditions are important for minimizing transient changes in the bulk temperature of the films. Unlike typical matrix isolation studies, our films are highly concentrated in chlorine (the principal absorber at $308 \mathrm{~nm}$ ). The heat capacity of the solid is low enough that even under our low fluence conditions, the bulk temperature of the films rises by an estimated $6 \mathrm{~K}$ after each pulse. ${ }^{10}$ Care is taken to make the samples sufficiently thin that the laser energy is absorbed nearly uniformly over the depth of the film (the samples usually transmit $60-80 \%$ of the $308 \mathrm{~nm}$ laser light).

Infrared spectra of the samples obtained after photolysis show that the absorption intensities of the reactant bands are diminished and new absorption bands of the reaction products appear. The products are identified by comparison with literature spectra and are confirmed in separate experiments by depositing some of the authentic compounds onto the sample window. By calibrating the integrated intensities of representative absorption bands with known amounts of samples deposited on the window, we are able to obtain absolute values of the reaction quantum yield (numbers of product molecules formed or reactants consumed per $308 \mathrm{~nm}$ photon absorbed by the samples).

The quantum yield measurements involve several steps. We measure the laser fluence with an absorbing disc calorimeter (Scientech Model 38-01) and the UV absorbance of the samples with a Cary 17 UV/VIS absorption spectrometer. The fraction of the total sample deposited in a $1 \mathrm{~cm}^{2}$ area at the center of the window is calculated to be $11 \%$ based on the geometry of the deposition tube ${ }^{10}$ (assuming a sticking coefficient of 1.0 for gas phase molecules impinging on the cold window). This number has been checked two ways. First, the absorbance of the samples at $308 \mathrm{~nm}$ is consistent with this figure if we take the molecular absorption cross section ${ }^{16}$ to be the same as the gas phase value. Secondly, photolysis of chlorine in some samples has been observed by Raman spectroscopy. ${ }^{13}$ The exponential 
decrease in chlorine concentration with increasing numbers of laser pulses is consistent with the gas phase absorption coefficient (assuming a unit quantum yield for dissociation). All three of these measurements (deposition geometry, absorption coefficient and dissociation yield) are consistent with $11( \pm 2) \%$ of the sample being deposited in $1 \mathrm{~cm}^{2}$ at the center of the sample window (the area probed by the IR spectrometer). For typical samples, the film thickness is approximately 5-10 $\mu \mathrm{m}$.

\section{RESULTS AND DISCUSSION}

\section{Chain reactions of chlorine with cyclopropane and cyclobutane}

Photochlorination of cyclopropane can occur by either a hydrogen abstraction mechanism yielding $\mathrm{HCl}$ and chlorocyclopropane products or by an addition mechanism forming 1,3-dichloropropane. The former is observed in gas phase studies, ${ }^{17}$ while both mechanisms occur in solution. ${ }^{18}$ In our solid state experiments, only the addition product is observed, as formed by Reactions (3) and (4).

$$
\begin{aligned}
& \mathrm{Cl} \cdot+\mathrm{c}-\mathrm{C}_{3} \mathrm{H}_{6} \longrightarrow \mathrm{ClCH}_{2} \mathrm{CH}_{2} \mathrm{CH}_{2} . \\
& \mathrm{ClCH}_{2} \mathrm{CH}_{2} \mathrm{CH}_{2} \cdot+\mathrm{Cl}_{2} \longrightarrow \mathrm{ClCH}_{2} \mathrm{CH}_{2} \mathrm{CH}_{2} \mathrm{Cl}+\mathrm{Cl} .
\end{aligned}
$$

The infrared absorption spectra of an equimolar mixture of $\mathrm{Cl}_{2}$ and cyclopropane before and after laser photolysis are presented in Figure 1. The IR bands of cyclopropane were characterized by using the spectral assignments of Breher et al. ${ }^{19}$ All bands were found to be slightly red-shifted $\left(5-10 \mathrm{~cm}^{-1}\right)$ relative to the gas phase values. Since chlorine is homonuclear it is transparent. Following irradiation the cyclopropane peaks are observed to decrease and new absorptions attributable to 1,3-dichloropropane appear. ${ }^{20,21}$ No evidence of any other products is observed. The assignments were confirmed and the intensities of the peaks calibrated by obtaining a spectrum of a sample containing a known quantity of the authentic compound. There are four possible conformers of 1,3-dichloropropane corresponding to various orientations of the chloromethyl groups. ${ }^{20}$ Interestingly, only the anti/anti (AA) conformer is formed in the reaction. Warming of the sample to a temperature near the melting point permits rotation about the $\mathrm{C}-\mathrm{C}$ bonds, and bands due to other conformers of 1,3dichloropropane are then observed. ${ }^{10}$ 


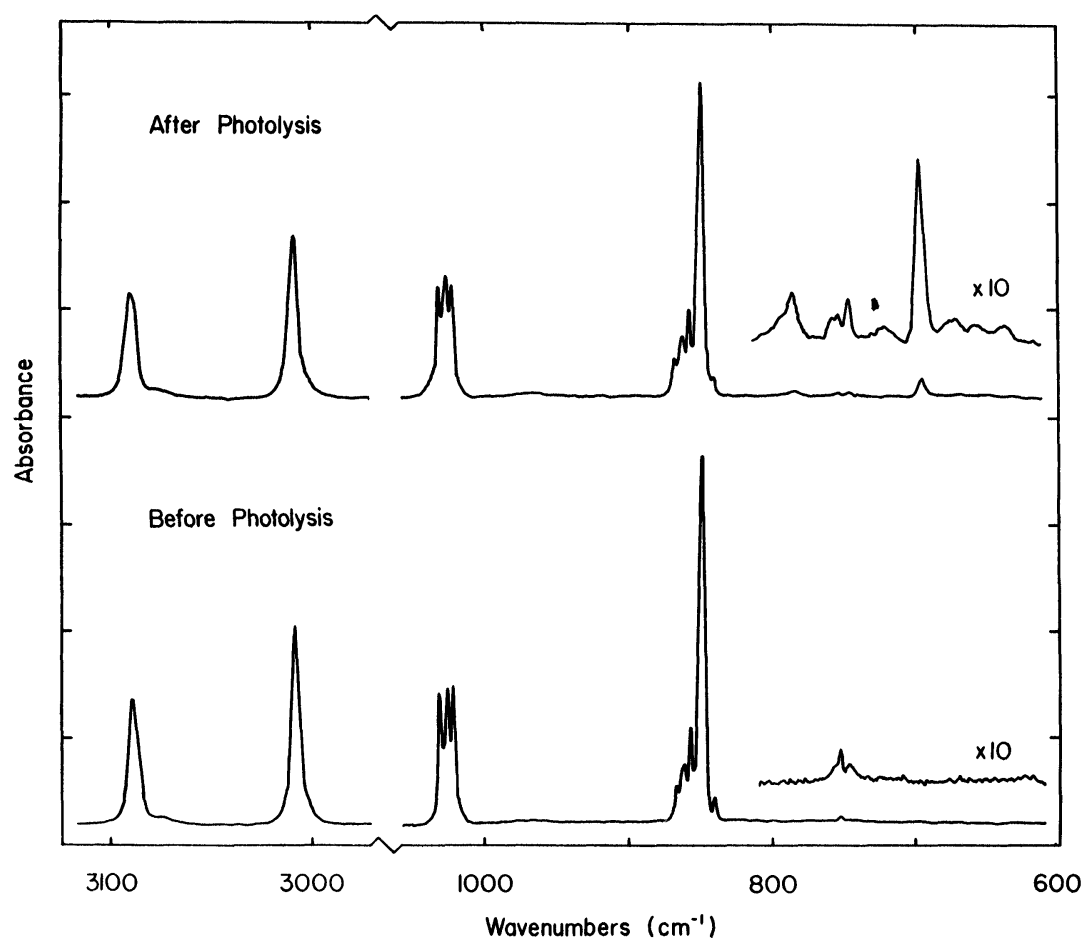

Figure 1 Infrared spectra of a 1:1 mixture of chlorine and cyclopropane before (lower trace) and after (upper trace) laser photolysis at $308 \mathrm{~nm}$. The sample was subjected to 35 laser pulses at an average fluence of $1.0 \mathrm{~mJ} / \mathrm{cm}^{2}$. The reaction product absorptions at 697 and $780 \mathrm{~cm}^{-1}$ are characteristic of the AA conformer of 1,3-dichloropropane (see text). Each division on the vertical scale represents 0.2 absorbance units whereas the magnified region is 0.02 units.

The best evidence that the reaction proceeds via a true chain reaction mechanism is the observation of quantum yields greater than unity. In fact, the yield for an equimolar mixture of chlorine and cyclopropane is measured to be $30 \pm 6$ for consumption of cyclopropane and $26 \pm 6$ for production of 1,3-dichloropropane. Photochemical quantum yields for several different relative concentrations of the two reagents are listed in Table I. There are two important observations to be made from these results. The first is that although the yields are much greater than 1.0 , they are small in comparison with typical chain lengths measured in the gas phase and in solution. The second 
Table I Photochemical quantum yields in mixtures of cyclopropane and chlorine at $77 \mathrm{~K}$ following $308 \mathrm{~nm}$ laser photolysis.

\begin{tabular}{llcc}
\hline $\mathrm{C}_{3} \mathrm{H}_{6}: \mathrm{Cl}_{2}{ }^{\mathrm{a}}$ & $\boldsymbol{x}^{\mathrm{b}}$ & Yield (reactants) $^{\mathrm{c}}$ & Yield (products) $^{\mathrm{d}}$ \\
\hline $0.2: 1$ & 0.83 & $2.6 \pm 0.6$ & $5.6 \pm 1.2$ \\
$0.5: 1$ & 0.67 & $7.6 \pm 1.6$ & $10.2 \pm 2.0$ \\
$1.0: 1$ & 0.50 & $30.0 \pm 6.0$ & $26.0 \pm 6.0$ \\
$2.0: 1$ & 0.33 & $16.8 \pm 3.4$ & $18.0 \pm 4.0$ \\
$5.0: 1$ & 0.17 & $7.0 \pm 1.2$ & $14.0 \pm 6.0$ \\
$9.0: 1$ & 0.10 & $6.2 \pm 3.0$ & $11.2 \pm 6.0$
\end{tabular}

${ }^{\text {a }}$ Relative pressures of reagents in sample prior to deposition.

b Mole fraction of chlorine in sample.

${ }^{c}$ Determined from diminution of cyclopropane absorbance at 3090 and $1425 \mathrm{~cm}^{-1}$.

d Determined from 1,3-dichloropropane absorbance at 780 and $697 \mathrm{~cm}^{-1}$.

observation is that the largest yields are obtained for samples in which the relative concentrations of the two reagents are equal.

We are now prepared to speculate on some of the details of the reaction mechanism in the solid state. Under low fluence conditions, the fraction of chlorine molecules dissociated in each pulse is approximately $10^{-4}$. On average, these molecules will be far apart in the solid, making radical recombination an unlikely mechanism for terminating the reaction. Instead, we consider a simple statistical model which involves the trapping of radicals in unreactive sites in the solid.

In the development of this model it is assumed that each free radical is surrounded by six neighboring sites which may be occupied by potential reaction partners. One of these is always occupied by 1,3dichloropropane formed in the previous step of the reaction. The probability that all five remaining sites are all occupied by $\mathrm{Cl}_{2}$ molecules is simply the $\mathrm{Cl}_{2}$ mole fraction raised to the fifth power. A chlorine atom in this situation is considered to be trapped, effectively terminating the reaction. The probability that the chlorine atom will successfully complete Reaction (3) is therefore

$$
P(3)=1-x^{5}
$$

where $x$ is the mole fraction of chlorine in the sample. Likewise, a chloropropyl radical is considered trapped if all five available sites around it are occupied by cyclopropane molecules. The probability of completing Reaction (4) is

$$
P(4)=1-(1-x)^{5} \text {. }
$$


Since both steps are required to form a single 1,3-dichloropropane product, the probability of successfully completing a single cycle is $P(3) P(4)$. The total number of product molecules formed per $\mathrm{Cl}$ atom generated by photolysis is simply the summation of $[P(3) P(4)]^{k}$ over the number of completed cycles $k$,

$$
\text { chain length }=\sum_{k=1}^{\infty}[P(3) P(4)]^{k}=P(3) P(4) /[1-P(3) P(4)] .
$$

To obtain the quantum yield, this result is multiplied by 2 since each photon generates two $\mathrm{Cl}$ atoms which (presumably) initiate separate chain reaction sequences. A comparison of this statistical prediction with the measured quantum yields in the lower panel of Figure 2 shows this model to be in qualitative agreement with experiment. There are significant differences between the observed and calculated yields, especially in samples which are rich in cyclopropane, but the overall behavior is consistent with the model. It should be noted that the calculated yields are not scaled to the experiment; absolute values are calculated directly from Eq. (7). The only adjustable parameter of the model is the choice for the number of nearest neighbors for each radical.

We have not been able to detect the infrared absorption bands of the radicals directly, presumably because the radical concentrations are small and many of the absorption bands overlap those of the products or reactants. However, we have found indirect evidence for the existence of trapped radicals by annealing the samples. This releases some of the radicals and causes more of the product to be formed. In experiments which utilize high laser fluence (up to about $35 \mathrm{~mJ} / \mathrm{cm}^{2}$ ) we have observed that the quantum yield for the second pulse can be higher than that for the first. This is because the second pulse not only dissociates $\mathrm{Cl}_{2}$ molecules, but also raises the temperature of the solid high enough to release some of the radicals trapped after the first pulse.

Similar experiments involving photochlorination of cyclobutane also offer interesting observations concerning the reaction mechanism in the solid state. This reaction occurs exclusively by an $\mathrm{H}$ atom abstraction mechanism depicted by Reactions (8) and (9).

$$
\begin{aligned}
& \mathrm{Cl} \cdot+\mathrm{c}-\mathrm{C}_{4} \mathrm{H}_{8} \longrightarrow \mathrm{HCl}+\mathrm{c}-\mathrm{C}_{4} \mathrm{H}_{7} \cdot \\
& \mathrm{c}-\mathrm{C}_{4} \mathrm{H}_{7} \cdot+\mathrm{Cl}_{2} \longrightarrow \mathrm{c}-\mathrm{C}_{4} \mathrm{H}_{7} \mathrm{Cl}+\mathrm{Cl} .
\end{aligned}
$$




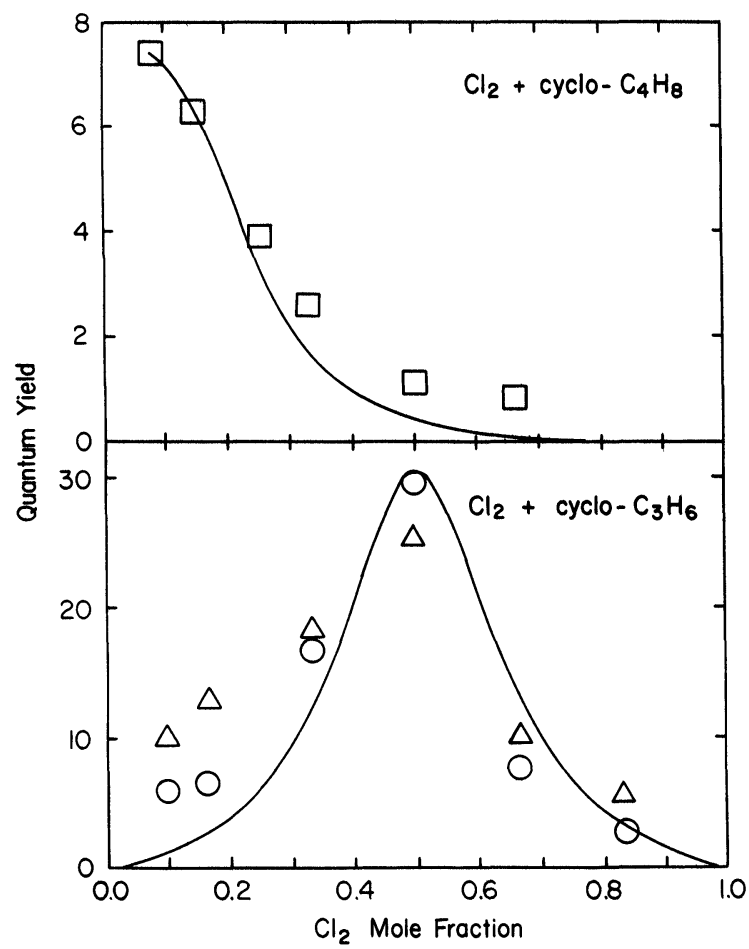

Figure 2 Plot of the photochemical quantum yield for mixtures of chlorine and cyclobutane (upper panel) and cyclopropane (lower panel) as a function of mole fraction of chlorine in unphotolyzed samples. In the upper panel, the squares represent experimental determinations based on chlorocyclobutane product formation. The solid curve is a prediction based on a computer simulation of the reaction kinetics which allows for $\mathrm{H}$ atom transfer from cyclobutane to cyclobutyl radicals as an intermediate step. In the lower panel, the triangles are based on formation of 1,3-dichloropropane whereas the circles are based on consumption of cyclopropane. The solid curve is calculated from Eq. (7) and is based on a simple statistical model of radical trapping.

The $\mathrm{C}-\mathrm{H}$ bond dissociation energy of cyclobutane is $41 \mathrm{~kJ} / \mathrm{mole}$ less than that of cyclopropane,$^{22}$ making the activation energy $\left(E_{a}\right)$ for the abstraction step low enough to compete favorably with ring opening. Knox and Nelson ${ }^{23}$ determined the $E_{a}$ for Reaction (8) to be $3.4 \mathrm{~kJ} /$ mole in the gas phase. In contrast, the $E_{a}$ for $\mathrm{H}$ atom abstraction from cyclopropane is $17.3 \mathrm{~kJ} / \mathrm{mole}$, which is high enough to effectively prevent the reaction from occurring on normal laboratory time scales at $77 \mathrm{~K}$. 
A somewhat curious observation in the cyclobutane system is that the quantum yield for formation of the chlorocyclobutane product is a maximum in samples which are rich in the hydrocarbon component. Yields for several different mixtures are presented in Table II and are represented graphically in the upper panel of Figure 2. In the statistical model presented above, we assumed that in order for reaction to occur, each free radical had to have at least one potential reaction partner among its six nearest neighbors. For chlorine/cyclobutane experiments in which the chlorine mole fraction is 0.08 , the probability that any cyclobutyl radical formed in Reaction (8) has a chlorine molecule in one of the five available sites is only 0.34 . The quantum yield predicted using Eq. (7) is only 0.52 and should increase with increasing chlorine concentration. The experimentally determined quantum yield is $7.4 \pm 0.8$ and has the opposite concentration dependence. Even if each radical could interact with 12 of its nearest neighbors (a practical upper limit), the predicted quantum yield is only 1.7 .

Table II Photochemical quantum yield for mixtures of chlorine and cyclobutane at $77 \mathrm{~K}$.

\begin{tabular}{lll}
\hline $\mathrm{C}_{4} \mathrm{H}_{8}: \mathrm{Cl}_{2}{ }^{\mathrm{a}}$ & $x^{\mathrm{b}}$ & Yield $^{\mathrm{c}}$ \\
\hline $0.5: 1$ & 0.67 & $0.8 \pm 0.3$ \\
$1.0: 1$ & 0.50 & $1.1 \pm 0.3$ \\
$2.0: 1$ & 0.33 & $2.6 \pm 0.5$ \\
$3.0: 1$ & 0.25 & $3.9 \pm 0.7$ \\
$5.2: 1$ & 0.16 & $6.2 \pm 1.1$ \\
$11.5: 1$ & 0.08 & $7.4 \pm 0.8$ \\
\hline
\end{tabular}

${ }^{a}$ Relative pressures of reagents prior to deposition.

${ }^{b}$ Mole fraction of chlorine in sample.

${ }^{c}$ Determined from chlorocyclobutane product absorptions.

The high yields and concentration dependence therefore suggest that cyclobutyl radicals are able to migrate over substantial distances in the solid in order to seek out molecular chlorine with which to react. Physical diffusion is out of the question since the temperature of the experiments $(77 \mathrm{~K})$ is far below the melting point of cyclobutane $(223 \mathrm{~K})$. A more likely explanation involves symmetric $\mathrm{H}$ atom transfer reactions between cyclobutane and cyclobutyl radicals, Reaction (10).

The idea that the mobility of a radical in solids involves, in some 


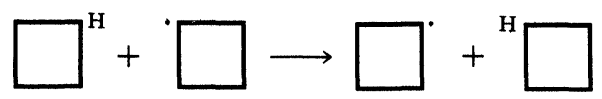

cases, the successive transfer (hopping) of a hydrogen atom was proposed by Dole and co-workers ${ }^{24}$ who observed the elimination of vinylidene groups in polyethylene following gamma irradiation. Additional evidence for a hydrogen hopping mechanism has recently been provided by Clough ${ }^{25}$ who observed H/D exchange in gamma irradiated tetracosane $\left(\mathrm{h}_{50} / \mathrm{d}_{50}\right)$ crystals. A drawback of the studies involving gamma radiolysis is that details of the initiation process are often not well understood. In our studies, photolysis at low fluence in the near ultraviolet virtually eliminates the possibility of forming ions or other high energy species in the solid which might complicate the analysis.

The curve shown in the upper panel of Figure 2 represents the prediction of a model of cyclobutane reactions based on the participation of $\mathrm{H}$ atom transfer reactions. The model assumes that any radical can react only with its six nearest neighbors. In samples rich in chlorine, $\mathrm{Cl}$ atom trapping is the dominant mechanism for stopping the reaction. However, in hydrocarbon-rich samples, the principal mechanism is radical recombination. Because of this, the concentration dependence of the quantum yield could not be solved analytically. Instead, the prediction in Figure 2 is based on a computer simulation of the reaction mechanism in which Reactions (8)-(10) as well as radical recombination processes are included. While the absolute values of the quantum yields obtained from the simulations are sensitive to details of the calculation, the general features of the concentration dependence are in agreement with experiment.

Based on our experimental results with propane and butanes (vide infra) as well as the computer simulations, we expect that radical recombination plays a substantial role in the reaction of chlorine with cyclobutane. True chain reactions must occur to at least some extent since the quantum yields are greater than 1.0 for hydrocarbon-rich samples. However, we have not yet found a reliable way to measure the chain length and thus cannot distinguish between cases in which product formation is due to a small number of very long chain reactions or a large number of short ones.

Future experiments are planned in which H/D exchange will be observed between cyclobutane and cyclobutane- $d_{8}$ in samples doped. 
with small amounts of chlorine. Quantitative analysis of the isotope effect for $\mathrm{H}$ vs $\mathrm{D}$ atom transfer will provide important clues to the role of quantum mechanical tunneling in solid state reactions.

\section{Photochlorination of propane and butane}

In contrast to the relatively high quantum yields observed for cyclopropane and cyclobutane, similar studies with straight chain hydrocarbons have afforded results which are much less spectacular. The quantum yield in the propane system, for example is $0.12 \pm 0.01$ for formation of 1-chloropropane and 2-chloropropane from an equimolar mixture of propane and chlorine. Although the participation of chain reaction processes cannot be definitively ruled out, the low quantum yield strongly suggests that radical recombination processes dominate the reaction mechanism. One way to visualize this is depicted in the upper panel of Figure 3. Reaction is initiated by photodissociation of molecular chlorine to two $\mathrm{Cl}$ atoms. The solid environment confines the atoms to a local cage ${ }^{26}$ which allows them to recombine to molecular chlorine. In a small fraction of these events, one of the $\mathrm{Cl}$ atoms may abstract an $\mathrm{H}$ atom from a nearby propane molecule forming $\mathrm{HCl}$. The propyl radical generated by this reaction may then recombine with the partner chlorine atom (which was trapped in the same cage) to form 1-chloropropane or 2-chloropropane depending on whether the initial $\mathrm{H}$ atom abstraction occurred at a primary or secondary position on the hydrocarbon. The two chloropropanes and $\mathrm{HCl}$ are observed in the infrared specra of irradiated samples of chlorine and propane and are the only reaction productions observed.

For the purposes of comparison, Figure 3 also depicts a proposed mechanism for the chain reaction of chlorine with cyclopropane. In this case, the ring opening step forms a chloropropyl radical in which the unpaired electron is spatially removed from the partner chlorine atom. We believe that the ring opening is therefore crucial to the separation of radical pairs and is largely responsible for the observation of chain reactions in the cyclopropane system. The observation that only the AA conformer of 1,3-dichloropropane is formed in the chain reaction provides detailed information about the ring opening step and the spatial relationships of the molecules during the course of reaction, as previously discussed. ${ }^{10}$ Although the role of radical recombination in the cyclopropane system cannot be ruled out, chain 


\section{Proposed reaction mechanism for low product yields: $\mathrm{Cl}_{2}+\mathrm{C}_{3} \mathrm{H}_{8}$}
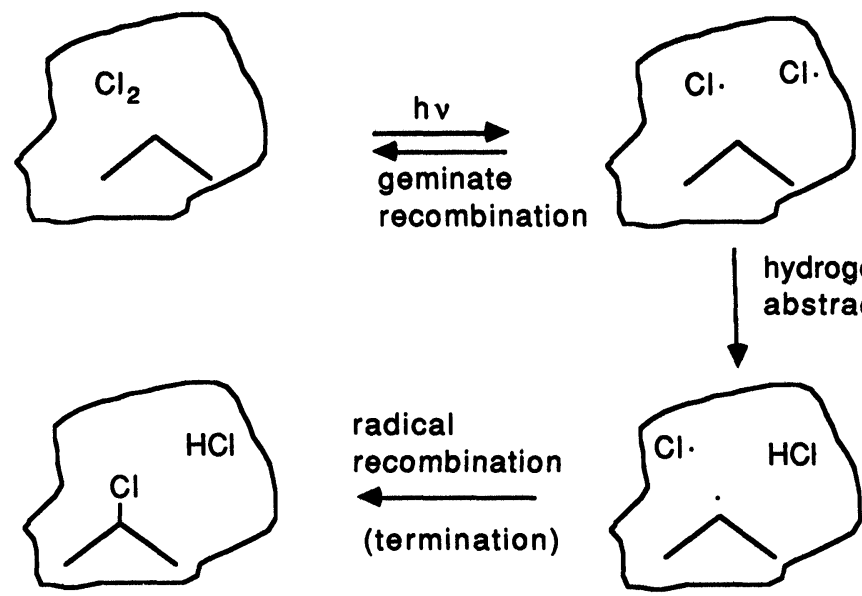

radical recombination (termination)

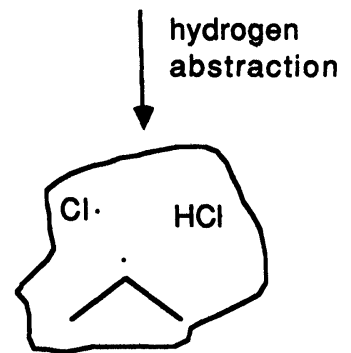

Proposed reaction mechanism for high product yields: $\mathrm{Cl}_{2}+$ cyclo- $\mathrm{C}_{3} \mathrm{H}_{6}$
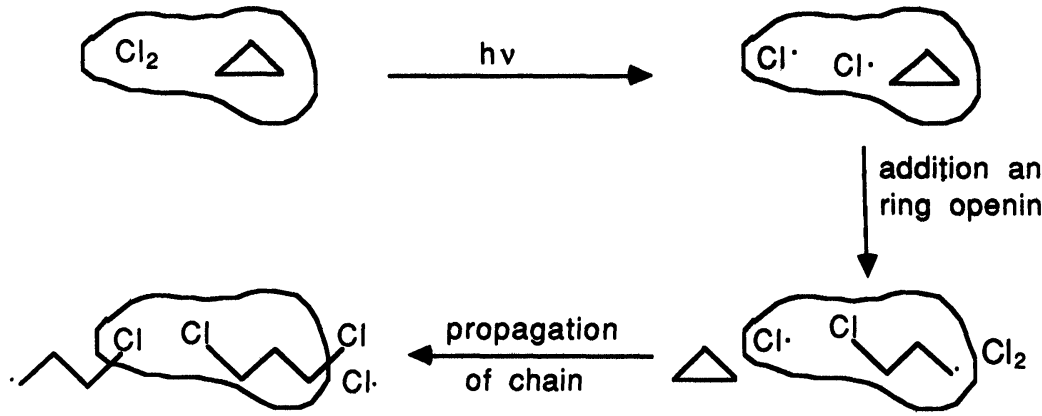

Figure 3 This is a pictorial representation of how mechanistic details of a reaction can be influenced by a solid-state environment. In the upper panel, a chlorine molecule and propane molecule are shown in some ill-defind site within the solid. Caging of the two chlorine atoms produced by laser photolysis allows efficient recombination of the radical pair, resulting in low quantum yields. In a small fraction of events, $\mathrm{H}$ atom abstraction forms $\mathrm{HCl}$. However, the resulting alkyl radical may recombine with the partner chlorine atom to generate the observed products. The proposed cyclopropane mechanism (shown in the lower panel) is qualitatively different because the ring opening step forms a chloropropyl radical which has its unpaired electron spatially removed from the partner chlorine atom. This orientation of the radical is inferred from a conformational analysis of the final reaction product (see reference 10). The solid prevents rotation of the radical, thereby inhibiting recombination processes. Each $\mathrm{Cl}$ atom may initiate separate chain reactions which propagate through the solid. 
reactions obviously play a major role in the reaction mechanism as this is the only reasonable way in which quantum yields greater than 1.0 can be rationalized.

An interesting aspect of the propane reaction system involves the selectivity of $\mathrm{H}$ atom abstraction by a chlorine atom at secondary vs. primary positions on the hydrocarbon. At $77 \mathrm{~K}$, we observed that the product branching ratio (2-chloropropane:1-chloropropane) is 5.6:1. When corrected for the fact that propane contains only one third as many secondary $\mathrm{H}$ atoms as primary, the relative reactivity is $17: 1$. The selectivity presumably arises from the lower activation energy for abstraction at the secondary site (in turn, reflecting the greater stability of the secondary radical). Interestingly, this ratio of products is exactly that which one would expect at $77 \mathrm{~K}$ based on the gas phase Arrhenius parameters determined by Knox and Nelson. ${ }^{23}$ The agreement may be fortuitous, but the $\mathrm{Cl}$ atoms nevertheless exhibit a remarkable degree of selectivity for attack at the secondary position of propane.

When experiments are conducted at lower temperatures,${ }^{11}$ the selectivity for formation of the lower energy product actually decreases. Below about $50 \mathrm{~K}$, the relative reactivity is approximately $2.5: 1$. The Arrhenius parameters ${ }^{23}$ predict the relative reactivity to be 2.3:1 in the limit of high temperature. We have therefore interpereted this observation in terms of reaction of hyperthermal $\mathrm{Cl}$ atoms with propane molecules. The energy of each $308 \mathrm{~nm}$ photon exceeds the bond dissociation energy of $\mathrm{Cl}_{2}$ by about $150 \mathrm{~kJ} /$ mole, so each $\mathrm{Cl}$ atom is born with a significant amount of excess translational energy. To the extent that this energy can be used to surmount the barrier to $\mathrm{H}$ atom abstraction, the selectivity for formation of 2-chloropropane should be diminished. Although the exact reasons for the onset of hot atom reactions below $50-70 \mathrm{~K}$ are unclear, we note that propane has a relatively low melting point $(85 \mathrm{~K})$. It is possible that thermalization of $\mathrm{Cl}$ atoms is more efficient in the slushy environment near the melting point compared to the more rigid solid below $50 \mathrm{~K}$.

Recent experiments conducted with n-butane and isobutane show similar behavior to propane. ${ }^{27}$ In each case the quantum yields are less than unity, making the participation of chain reaction steps unlikely. Also, the butane reactions exhibit a marked preference for formation of the lower energy reaction product when the sample is near its melting point. As in the case of propane, reducing the photolysis 
temperature reduces this selectivity, consistent with the onset of hot $\mathrm{Cl}$ atom reactions.

\section{CONCLUSIONS AND PROGNOSIS FOR FUTURE STUDIES}

Our investigations of free radical chain reactions have provided insight to mechanistic details of chemistry which occurs in amorphous solids. We have noted that in many cases, the classical cage effects results in efficient recombination of radical pairs produced by laser photolysis. However, this is not always the case. For example, ring opening of cyclopropane provides a mechanism for separating radicals. This inhibits recombination and promotes the onset of true free radical reactions in the solid.

Reactions of chlorine with acrylic hydrocarbons provides the opportunity to study the selectivity of $\mathrm{H}$ atom abstraction from primary, secondary and tertiary sites. In all cases studied so far, we have observed that near the melting point of the solid, selectivity of attack can be very high and is generally in agreement with the predictions of gas phase Arrhenius parameters. The clear implication is that under these conditions, $\mathrm{Cl}$ atoms are thermalized to the characteristic temperature of the environment on a time scale which is fast compared with reaction. At lower temperatures, this selectivity decreases and the results are consistent with reactions of hot $\mathrm{Cl}$ atoms generated by photolysis of $\mathrm{Cl}_{2}$. There is still much that we do not understand about the dynamics of energy transfer and chemical reactions in amorphous solids, and the field remains ripe for new investigations.

A variation of our chain reaction scheme involves laser-initiated polymerization of organic molecules. We have recently begun investigating reactions of chlorine with formaldehyde and find that polymerization is easily induced at temperatures as low as $10 \mathrm{~K}$. Details of the reaction mechanism will be presented in a forthcoming paper. ${ }^{13}$

Another promising area of research involves $\mathrm{H}$ atom transfer reactions in the solid. Particularly attractive is the possibility of characterizing the role of quantum mechanical tunneling by quantitative analysis of $\mathrm{H} / \mathrm{D}$ isotope effects. It may ultimately be possible to observe these reactions in real time and thus obtain rate coefficients for solid state reactions. 


\section{Acknowledgements}

The authors gratefully acknowledge the assistance of Mr. Edward Mansueto, who performed several of the experiments in the cyclopropane and propane systems. This research was supported by the U.S. Air Force Astronautics Laboratory under contract No. F04611-87-0023. Partial support was supplied by the Office of Naval Research under contract No. N0014-86-K-0710 through the University of Utah Laser Institute.

\section{References}

1. V. Ramamurthy and K. Venkatesan, Chem. Rev. 87, 433 (1987).

2. J. M. McBride, B. E. Segmuller, M. D. Hollingsworth, D. E. Mills and B. A. Weber, Science 234, 830 (1986).

3. R. Jankowiak, G. J. Small and B. Ries, Chem. Phys. 118, 223 (1987).

4. K. U. Ingold, Pure and Appl. Chem. 56, 1767 (1984).

5. A. Blumen, G. Zumofen and J. Klafter, Ber. Bunsenges. Phys. Chem. 90, 1048 (1986).

6. H. Ehrenreich, Science 235, 1029 (1987).

7. A. H. Walton, Structure and Properties of Amorphous Polymers (Elsevier Scientific, Amsterdam, 1980).

8. V. I. Goldanski, Ann. Rev. Phys. Chem. 27, 85 (1976).

9. R. Elber and M. Karplus, Science 235, 318 (1987).

10. A. J. Sedlacek, E. S. Mansueto and C. A. Wight, J. Am. Chem. Soc. 109, 6223 (1987).

11. A. J. Sedlacek and C. A. Wight, J. Phys. Chem., 92, 2821 (1988).

12. A. J. Sedlacek and C. A. Wight, J. Chem. Phys., 88, 2847 (1988).

13. E. S. Mansueto, C.-Y. Ju and C. A. Wight, J. Chem. Phys., submitted.

14. W. A. Pryor, Free Radicals (McGraw-Hill, New York, 1966), series in Advanced Chemistry.

15. C. Walling, Free Radicals in Solution (Wiley, New York, 1957).

16. H. Okabe, Photochemistry of Small Molecules (Wiley-Interscience, New York, 1978).

17. J. D. Roberts and P. H. Dirstine, J. Am. Chem. Soc. 67, 1281 (1945).

18. C. Walling and P. S. Fredericks, J. Am. Chem. Soc. 84, 3326 (1962).

19. C. Breher, E. Krikorian, J. Blane and R. S. Halford, J. Chem. Phys. 35, 1097 (1961).

20. J. Thorbjørnsrud, O. H. Ellostad, P. Klaboe and T. Torgrimsen, J. Mol. Struct. 15, 61 (1973).

21. J. K. Brown and N. Sheppard, Proc. Roy. Soc. (London) A231, 555 (1955).

22. D. F. McMillen and D. M. Golden, Ann. Rev. Phys. Chem. 33, 493 (1982).

23. J. H. Knox and R. L. Nelson, Trans. Faraday Soc. 55, 937 (1959).

24. M. Dole and C. D. Kneeling, J. Am. Chem. Soc. 75, 6082 (1953).

25. R. Clough, J. Chem. Phys. 87, 1588 (1987).

26. M. D. Cohen, Agnew Chem. Internat. Edit. 14, 386 (1975).

27. A. J. Sedlacek and C. A. Wight, unpublished. 